immoral acts (i.e., violations of obligation) as more objective, agreed upon by others, and true than beliefs about moral acts (i.e., fulfillments of obligation; Goodwin \& Darley 2012). On the whole, then, proscriptive obligations appear to have more psychological weight than prescriptive obligations.

It may be possible to reconcile these asymmetries within Tomasello's theoretical framework - for example, it may be that proscriptive obligations entail a greater sense of "we" in their formation and enforcement - but more work will be necessary to assess these possibilities.

Additionally, the process by which observers judge violations of obligation and fulfillments of obligation also appears to differ. For example, immoral, obligation-violating acts tend to elicit more causal attribution and counterfactual thinking than moral, obligation-fulfilling acts (Bohner et al. 1998; Bostyn \& Roets 2016; Roese \& Olson 1997). In addition, although agent intentionality influences the perceived morality and immorality of both obligation-fulfilling acts and obligation-violating acts, intentionality appears to matter more for evaluating blameworthy acts than praiseworthy acts (Guglielmo \& Malle 2019; Ohtsubo 2007; Pizarro et al. 2003). For example, both adults and children judge unintended, obligation-violating "side effects" (of a person's actions) to be blameworthy. However, they fail to judge unintended, obligation-upholding side effects to be equally praiseworthy (Knobe 2003a; 2003b; Leslie et al. 2006). People also more readily incorporate the magnitude of the consequences of proscriptive obligations than prescriptive obligations into their judgments of the agent (Gneezy \& Epley 2014; Klein \& Epley 2014). Together, this research suggests that judgments of proscriptive obligations incorporate factors related to causality, intentionality, and consequences more than do judgments of prescriptive obligations.

Importantly, research suggests that even young children have an awareness of this distinction between prescriptive and proscriptive violations. For example, children have been shown to exhibit better memory for negative, obligation-violating individuals than positive, obligation-fulfilling individuals (Barclay \& Lalumiere 2006; Kinzler \& Shutts 2008). Additionally, children as young as 14 months have greater difficulty following do's than don'ts (Kochanska et al. 2001). These findings suggest that these asymmetries do not simply reflect "second step" (sect. 2, para. 2) differences attributable to culture-specific learning, but rather emerge at an earlier ontogenetic stage.

These differences in judgments of prescriptive and proscriptive obligations suggest that there are important nuances to how developing humans learn about the moral obligations of their group and culture - and that the processes by which we learn about, represent, and evaluate prescriptive obligations (e.g., to obey our elders) may be different from those of proscriptive obligations (e.g., to not harm others).

Additionally, there is also evidence that the relevant weight accorded to each of these forms of obligation can differ as a function of the specific context in which they are embedded. For example, these two broad classes of moral obligation also seem to operate somewhat distinctly across intergroup boundaries. This appears to be an important caveat to Tomasello's claim that obligation "applies to, and only to, one's cultural compatriots" (sect. 2.2, para. 2). Whereas we agree that this claim generally holds true regarding prescriptive obligations (e.g., there is little expectation that one will help members of cultural outgroups), proscriptive obligations seem to be more common across intergroup boundaries (e.g., the obligation not to willingly harm members of cultural outgroups).
One apparent example of these asymmetries is the (rapidly growing) number of public apologies made by leaders of majority (racial/ethnic, religious, and/or cultural) groups for past injustices to minority groups - apologies which, almost without exception, center on violations of proscriptive obligations not to harm, rather than violations of prescriptive obligations (Blatz et al. 2009; Lazare 2004).

As Tomasello suggests, these cross-group apologies may indicate "an expanded sense of all of humanity as in one's in-group moral community" (sect. 2.2, para. 2). However, his account does not explain these apparent asymmetries in how prescriptive and proscriptive obligations operate across group boundaries.

We suggest that these prescriptive/proscriptive asymmetries may offer Tomasello a promising opportunity to refine his theory of moral obligation. These differences in how observers evaluate proscriptive and prescriptive obligations suggest that there may be different forms of obligation, each utilizing distinct psychological processes. At the very least, different moral obligations appear to engender different responses when they are upheld or violated. What psychologically distinguishes prescriptive obligations from proscriptive obligations? How could such differences emerge? For a complete account of the psychology of moral obligations, these questions ought to be addressed.

\section{Obligations to whom, obligations to what? A philosophical perspective on the objects of our obligations}

\section{Kati Kish Bar-On ()}

The Cohn Institute for the History and Philosophy of Science and Ideas, Tel Aviv University, Tel Aviv Yafo, Israel, 6997801.

katikish@gmail.com http://telaviv.academia.edu/KatiKish

doi:10.1017/S0140525X19002395, e58

\begin{abstract}
Tomasello strives to understand the underlying psychology behind the human sense of obligation, but he only addresses a specific kind of obligation: to other human beings. We argue that in order to account for the psychological underpinning of human behavior, one should also consider people's sense of commitment to non-human entities, such as ideals, values, and moral principles.
\end{abstract}

Tomasello presents a compelling analysis of the motivating force behind humans' sense of obligation, focusing on the intersubjective structure of an obligation. However, obligations are not always immersed in agreements only between individuals. Occasionally, they are promises we make to ourselves, our commitments to a particular ideal of living or a moral value - entities that Tomasello does not discuss. These two different types of obligations can together explain individuals' behavior. If Bob is impudent to Charlie and Alice condemns Bob's behavior, Alice's criticism may derive from her heartfelt commitment to a certain principle of proper behavior to which she holds everyone accountable; she does not necessarily think that Bob has a sense of 
obligation to Charlie or anyone else. She holds Bob accountable to the standard itself, not to other people. Alice cares about the ideal of proper behavior; therefore, it is important to her that people follow a certain behavioral standard when engaging with others.

The philosopher Harry Frankfurt argued that human behavior is shaped by the things we care about (Frankfurt 1982). Although ethical considerations regulate our relations with other people (using moral obligations), we often do not find the requirements of ethics to be the only things we care about. Even people with a strong sense of moral obligations to others can care equally about ideals such as being loyal to a family tradition or can devote themselves to ecological principles of fighting climate change (Frankfurt 2006).

Some people find moral obligations to be their most important obligations, above all other commitments. Others may choose to intentionally violate a moral obligation to another person not because there is a stronger moral obligation to which they are committed, but rather because they consider a certain value, ideal, or course of action to be more important to them than meeting the demands of moral obligations to others. Frankfurt's work draws our attention to the possibility that a unanimous hierarchical scale of obligations (where morality is superior to all other commitments) may not exist. An individual's sense of obligation to other people as well as to specific values or standards varies not only between people but also between situations: A person can choose to fulfill his obligation to another individual in one situation, but in a different circumstance he might choose to obey his commitment to a certain value or ideal over a moral obligation to a person.

Frankfurt has addressed this type of commitment as an integral part of one's inner identity, which serves as the reason and motivation for one's actions. He writes:

A person who cares about something is, as it were, invested in it. He identifies himself with what he cares about in the sense that he makes himself vulnerable to losses and susceptible to benefits depending upon whether what he cares about is diminished or enhanced. Thus he concerns himself with what concerns it, giving particular attention to such things and directing his behavior accordingly. (Frankfurt 1988, p. 83)

People whose behavior is governed by their commitments to the ideals they care about rather than by their moral obligations to others may be considered self-centered or judged as a person who lacks empathy. However, as Frankfurt has pointed out, the structure of people's personality is more complex than a onedimensional scale of morality on one end and self-interest on the other. He argued that persons may feel committed to certain cultural or religious ideals that derive neither from moral nor egoistic considerations and may pursue those nonmoral ideals without considering their own personal goals (Frankfurt 2004).

Even though Frankfurt challenged the widely accepted authoritative nature of moral obligations, he did not claim that our relationships with other people or the moral requirements for maintaining those relationships are not important to us. He offered the possibility that other types of commitments may count as heavily or even more heavily with us, and he held that one should not assume that moral obligations always override them. For Frankfurt, what guides our behavior is not our moral or nonmoral obligations per se, but rather our attitude toward them: how much we care about these obligations and how important it is for us to carry them out.

According to Frankfurt, the fact that one cares about a certain thing is constituted by a complex set of cognitive dispositions, but he did not account for the interpersonal factors that affect those dispositions. The role of an individual's social environment and group expectations in shaping human behavior is, to some extent, left out of Frankfurt's discussion. To fill the lacuna, let us return to Alice's disapproval of Bob's behavior: Even if Alice profoundly cares about a certain ideal of proper behavior, why should she hold Bob accountable to the same ideal? What urges her to condemn Bob's acts? Whereas Frankfurt did not engage in this type of question, Tomasello considers the societal aspects that are missing from Frankfurt's account. In Tomasello's view, the origins of Alice's expectations may derive from her view of herself, Bob, and Charlie, as members of the same cultural group. As such, all group members are obligated to conform to the group's ways or, if not, they must provide an explanation for their deviation. The object of Alice's sense of obligation may be a standard rather than a person, but in any case, her cultural identity and group affiliation will always play a significant role in the process of cultivating the things she cares about and her attitude toward transgression.

To conclude, human or non-human entities can be the objects of our feelings of obligation, and those, in turn, influence and shape our behavior. My intention in this commentary was to point toward an enhanced psychological account of humans' sense of obligation, including the approaches of both Frankfurt and Tomasello. Each theory portrays only part of the picture; together, they offer a more comprehensive account of individuals' feelings of obligation and the objects of those feelings.

\section{Children's everyday moral conversation speaks to the emergence of obligation}

\section{Karen Bartsch (1)}

Psychology Department, Wyoming University, Laramie, WY 82071-3415. bartsch@uwyo.edu http://www.uwyo.edu/psychology/faculty/bartsch.html

\section{doi:10.1017/S0140525X19002358, e59}

\section{Abstract}

For Tomasello's proposed ontology of the human sense of moral obligation, observations of early moral language may provide useful evidence complementary to that afforded by experimental research. Extant reports of children's everyday moral talk reveal patterns of participation and content that accord with the proposal and hint at extensions addressing individual differences.

In his account of the ontogeny of our human sense of moral obligation, Tomasello pinpoints its origin in early joint intentional action and describes two developments in the preschool years: the first regarding interpersonal obligation between collaborative partners and the second regarding norm-based morality within a cultural group. Tomasello invokes extensive support from cleverly designed experimental studies of young children's behavior (e.g., Gräfenhain et al. 2009; Rekers et al. 2011), which have the dual virtues of bypassing young children's verbal limitations and facilitating cross-species comparisons. But Tomasello's 